Document downloaded from:

http://hdl.handle.net/10251/44703

This paper must be cited as:

Lopez-Solano, J.; Rodriguez-Hernandez, P.; Muñoz, A.; Santamaria-Perez, D. et al.(2011). High-pressure theoretical and experimental study of HgWO4. High Pressure Research. 31(1):58-63. doi:10.1080/08957959.2010.521735.

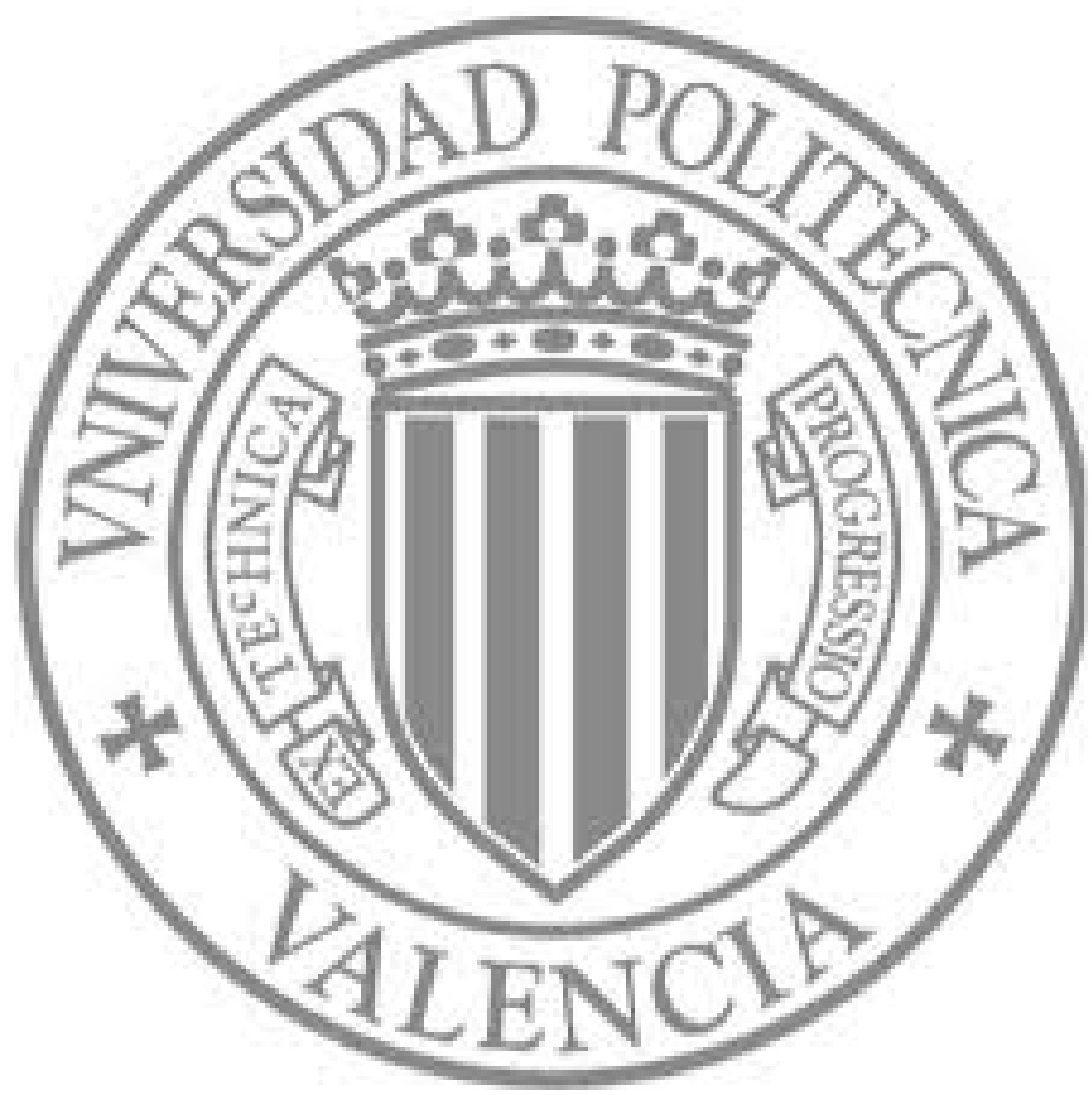

The final publication is available at

http://www.tandfonline.com/doi/abs/10.1080/08957959.2010.521735\#.Uo3ol9lz2Xw

Copyright Taylor \& Francis 
EHPRG Proceedings for 2010

\title{
High-pressure theoretical and experimental study of $\mathrm{HgWO}_{4}$
}

J. López-Solano, ${ }^{a^{*}}$ P. Rodríguez-Hernández, ${ }^{\mathrm{a}}$ A. Muñoz, ${ }^{\mathrm{a}}$ D. Santamaría-Pérez, ${ }^{\mathrm{b}}$ F.J. Manjón, ${ }^{\mathrm{c}}$ S. Ray, ${ }^{\mathrm{c}}$ O. Gomis, ${ }^{\mathrm{d}}$ M. Mollar, ${ }^{\mathrm{c}}$ V. Panchal, ${ }^{\mathrm{e}}$ and D. Errandonea ${ }^{\mathrm{e}}$

${ }^{a}$ MALTA Team - Departamento de Fisica Fundamental II, Universidad de La Laguna, 38205, La Laguna, Spain; ${ }^{\mathrm{b}}$ Departamento de Química Física I - MALTA Consolider Team, Universidad Complutense de Madrid, Avda. Complutense s/n, 28040 Madrid, Spain; ${ }^{c}$ Instituto de Diseño para la Fabricación y Producción Automatizada - MALTA Consolider Team, Universitat Politècnica de València, 46022 València, Spain; ${ }^{d}$ Centro de Tecnologías Físicas MALTA Consolider Team, Universitat Politècnica de València, 46022 València, Spain; ${ }^{e}$ Instituto de Ciencia de Materiales de la Universitat de València y Fundación General de la Universitat de València - MALTA Consolider Team, 46100 Burjassot (València), Spain

\begin{abstract}
$\mathrm{HgWO}_{4}$ at ambient pressure is characterized using a combination of $a b$ initio calculations, X-ray diffraction and Raman scattering measurements. The effect of low pressure and temperature on the structural stability is analyzed. Extending our ab initio study to the range of higher pressures, a sequence of stable phases up to 30 $\mathrm{GPa}$ is proposed.
\end{abstract}

Keywords: phase transitions; tungstates; ab initio calculations; Raman scattering; X-ray diffraction

Title Page Footnote:

*Corresponding author. Email: javierl@marengo.dfis.ull.es

\section{Introduction}

In spite of the interest $A B \mathrm{O}_{4}$ compounds have attracted in recent years, the pressure behaviour of mercury tungstate remains largely unknown. It is however a rather interesting compound, since it does not crystallize in the scheelite $\left(\mathrm{CaWO}_{4}\right.$-type, space group $[\mathrm{SG}]$ no. $\left.88, I 2 / a, Z=4\right)$ nor in the wolframite $\left(\mathrm{NiWO}_{4-}\right.$ type, SG no. 12, $P 2 / c, Z=2$ ) structures, the two most usual ambient-pressure structures for tungstates [1]. The $\mathrm{HgWO}_{4}$-type ( $\mathrm{SG}$ no. 15, $C 2 / c, Z=4$ ) structure has some similarities with the wolframite, but in particular the 
cationic environments are different, as reflected by the coordinations: $[6,6]$ for wolframite and $[6+2,6]$ for $\mathrm{HgWO}_{4}$-type, where the first number in brackets is the oxygen coordination of the $\mathrm{Hg}$ cation, and the second, that of the $\mathrm{W}$. Thus, the sequence of stable phases under pressure of $\mathrm{HgWO}_{4}$ can be expected to have notable differences with respect to the ones proposed for compounds with the scheelite or wolframite structure, scheelite $\rightarrow$ fergusonite $\rightarrow \mathrm{BaWO}_{4}$-II-type [1] and wolframite $\rightarrow \mathrm{CuWO}_{4}$-type $\rightarrow$ Cmca [2-4] respectively.

In this paper, we study the pressure behaviour of $\mathrm{HgWO}_{4}$. Although we include some results of our Xray diffraction and Raman scattering measurements, we will focus on our theoretical ab initio calculations. Further details of our experimental work can be found in [5].

\section{Method}

All the ab initio calculations presented in this work have been performed with the VASP [6,7] code, which works within the density functional theory framework, using plane-waves basis and pseudopotentials. Projector augmented-wave [8] pseudopotentials and the recently proposed PBEsol [9] approximation for the exchange and correlation energy have been adopted. The latter is a GGA approximation tuned to produce a good description of the structural equilibrium properties of solids and surfaces.

An energy cutoff of $530 \mathrm{eV}$ for the plane-waves basis and dense Monkhorst-Pack [10] reciprocal space grids ensure an energy convergence of $1 \mathrm{meV}$ per formula unit. For each structure and volume considered, a full optimization of the lattice parameters and atomic positions was performed until the components of the stress tensor differed from the isotropic diagonal form in less than $0.1 \mathrm{GPa}$, and the maximum atomic force was lower than $0.001 \mathrm{eV} / \AA^{3}$.

The energy and volume data were fitted with a $4^{\text {th }}$ order Birch-Murnaghan equation of state to obtain the pressure, equilibrium volume $\left(V_{0}\right)$, bulk modulus $\left(B_{0}\right)$ and the first derivative of the latter $\left(B_{0}^{\prime}\right)$. From the values of energy, volume and pressure, the enthalpy of each structure was calculated to determine the most stable phase amongst the ones considered at each pressure and zero temperature (zero-point motion effects were also not included). The Gibbs free energy was calculated within the quasi-harmonic Debye approximation with the GIBBS [11] code to estimate the effect of temperature on the $\mathrm{HgWO}_{4-}$ type $\rightarrow$ wolframite transition. Phonon frequencies at the $\Gamma$ point of the $\mathrm{HgWO}_{4}$-type structure were calculated using the small displacements method [12].

\section{Results and discussion}


Figure 1 shows the energy as a function of volume curves for all the structures considered as possible stable phases of $\mathrm{HgWO}_{4}$. Among these structures, the calculations find the $\mathrm{HgWO}_{4}$-type as the lowest-energy phase at zero pressure, in agreement with the experimental observations. The calculated values of $V_{0}, B_{0}$, and $B_{0}^{\prime}$ are respectively $81.61 \AA^{3}$ per formula unit, $60.6 \mathrm{GPa}$, and 8.9 , which are in good agreement with the experimental values of $81.01 \AA^{3}, 72.7 \mathrm{GPa}$, and 5.1. In particular note that the PBEsol approximation overestimates $V_{0}$ by less than $1 \%$, much lower than the usual 5\% produced by other GGA approximations.

In Table 1, calculated Raman frequencies are presented together with the data from our Raman experiments. The overall agreement is quite reasonable, with a small underestimation of the theoretical values with respect to the experimental ones which has been related to the GGA approximation [13]. X-ray diffraction patterns can also be indexed with a $\mathrm{HgWO}_{4}$-type structural model (see Figure 2.(a) in [5]). Thus, the ambient-pressure phase of $\mathrm{HgWO}_{4}$ is unequivocally determined.

Upon increase of pressure, the wolframite structure becomes more stable than the $\mathrm{HgWO}_{4}$-type, as shown by the free energy as a function of pressure curves of Figure 2. As pointed out in [14], both structures are very similar. In the $I 2 / a$ setting, the $\mathrm{HgWO}_{4}$-type structure features lattice parameters very close to those of a wolframite $P 2 / c$ supercell doubled along its $c$ axis, as shown in Figure 3. The atomic positions are also similar, the most noticeable difference being in the stacking of the oxygens [14], which produces rather different environments around both cations. Despite all these similarities, which justify their proximity in energy, it should be stressed that in our calculations the two structures are markedly different.

It should also be noted that in our experiments at ambient temperature we didn't found indications of the wolframite structure up to the maximum pressure reached of $16 \mathrm{GPa}$ for X-ray diffraction experiments and $25 \mathrm{GPa}$ for Raman scattering measurements (although the quality of the Raman spectra above 16 GPa is poor [5]). To estimate the effect of the temperature on these energetically close phases, we have calculated the Gibbs free energy of the $\mathrm{HgWO}_{4}$-type and wolframite structures at $300 \mathrm{~K}$ within the Debye approximation. The difference in free energy between both phases is marked by the discontinuous line of Figure 2.(b). As shown, temperature makes the wolframite phase more unfavourable with respect to the $\mathrm{HgWO}_{4}$-type, and the transition pressure is increased from $\sim 2 \mathrm{GPa}$ at $0 \mathrm{~K}$ to $\sim 11 \mathrm{GPa}$ at $300 \mathrm{~K}$. This pressure is rather close to the maximum reached in experiments, where measurements are of lower quality, and this may justify the lack of experimental observations of the wolframite phase.

As a final comment with regard to the stability at lower pressures, we note that in the present calculations a full optimization of the lattice and atomic parameters of the triclinic $\mathrm{CuWO}_{4}$-type (SG no. 2, $P$ - 
$1, Z=2)$ structure have lead to the wolframite structure. This is in marked contrast to the situation found in the $\mathrm{Mg}, \mathrm{Zn}$, and $\mathrm{Cd}$ tungstates [2-4]. Also, the fergusonite ( $\mathrm{YNbO}_{4}$-type, SG no. 15, I2/a, Z=4) structure has been found to be identical to the scheelite.

Extending our $a b$ initio study to higher pressures, we have considered several candidates for stable phases: $\mathrm{BaWO}_{4}$-II-type (SG no. $14, P 2_{1} / n, Z=8$ ), Cmca (SG no. $64, C m c a, Z=8$ ), $\mathrm{BaZnCl}_{4}$-type (SG no. 60, $P b c n, Z=4$ ), $\mathrm{LaTaO}_{4}$-type ( $\mathrm{SG}$ no. 14, $P 2 / c, Z=4$ ), $\mathrm{CaUO}_{4}$-type (SG no. 166, $R$-3m, $Z=1$ ), $\mathrm{BaMnF}_{4}$-type (SG no. 36, $A 2_{1} a m, Z=4$ ), and $\mathrm{SrUO}_{4}$-type (SG no. 57, $\mathrm{Pbcm}, Z=4$ ) structures. Some of these structures have been found to be energetically competitive in previous works and all are likely high-pressure phases on the basis of crystal-chemical considerations [1]. Although ambient temperature can affect energetically close phases at low pressures, it is unlikely that it will completely modify the sequence of stable phases under high pressure, and thus we have performed only zero-temperature calculations. As shown in Figure 2.(b), the $\mathrm{BaWO}_{4}$-IItype structure becomes the most stable after the wolframite and $\mathrm{HgWO}_{4}$-type ones. Increasing the pressure further, the $\mathrm{BaWO}_{4}$-II-type structure becomes unstable with respect to the Cmca. In these two first order transitions, the coordination changes first from $[6+2,6]$ to $[8,6]$, and then to $[11,6+1]$. The sequence $\mathrm{HgWO}_{4-}$ type $\rightarrow \mathrm{BaWO}_{4}$-II-type $\rightarrow$ Cmca of transitions under pressure bears similarities to the sequences of both the scheelite and the wolframite compounds.

\section{Concluding remarks}

The ab initio and experimental results presented in this work for the stability at low pressure and low temperature are in agreement, and show that the $\mathrm{HgWO}_{4}$-type is the stable phase in these conditions. At high pressure, the $\mathrm{BaWO}_{4}$-II-type and Cmca phases found stable in our ab initio calculations have been reported in

previous works on other $A B \mathrm{O}_{4}$ compounds. Further experimental work at high pressure would be needed to confirm the high pressure behaviour of $\mathrm{HgWO}_{4}$.

\section{Acknowledgments}

We thank J.M. Menéndez for his help in the use of the GIBBS code. This work has been supported by the Spanish MEC under Projects MAT2007-65990-C03-01/03 and CSD-2007-00045, and by the "Vicerrectorado de Innovación y Desarrollo de la UPV" (PAID-05-2009 through project UPV2010-0096). We gratefully acknowledge computational time provided by the "Red Española de Supercomputación" at the supercomputer "Atlante".

\section{References}


[1] F.J. Manjón, and D. Errandonea, Phys. Status Solidi B 246, 9 (2009).

[2] D. Errandonea, F.J. Manjón, N. Garro, P. Rodríguez-Hernández, S. Radescu, A. Mujica, A. Muñoz, and C.Y. Tu, Phys. Rev. B 78, 054116 (2008).

[3] J. Ruiz-Fuertes, S. López-Moreno, D. Errandonea, J. Pellicer-Porres, R. Lacomba-Perales, A. Segura, P. Rodríguez-Hernández, A. Muñoz, A.H. Romero, and J. González, J. Appl. Phys. 107, 083506 (2010).

[4] R. Lacomba-Perales, D. Errandonea, D. Martínez-García, P. Rodríguez-Hernández, S. Radescu, A. Mújica, A. Muñoz, J.C. Chervin, and A. Polian, Phys. Rev. B 79, 094105 (2009).

[5] F.J. Manjón, J. López-Solano, S. Ray, O. Gomis, D. Santamaría-Pérez, M. Mollar, V. Panchal, D.

Errandonea, P. Rodríguez-Hernández, and A. Muñoz, accepted in Phys. Rev. B (2010).

[6] G. Kresse, and J. Hafner, Phys. Rev. B 47, 558 (1993).

[7] G. Kresse, and J. Furthmüller, Phys. Rev. B 54, 11169 (1996).

[8] P. E. Blöchl, Phys. Rev. B 50, 17953 (1994).

[9] J.P. Perdew, A Ruzsinsky, G.I. Csonka, O.A. Vydrov, G.E. Scuseria, L.A. Constantin, X. Zhou, and K. Burke, Phys. Rev. Lett. 100, 136406 (2008).

[10] H.J. Monkhorst and J. D. Pack, Phys. Rev. B 13, 5188 (1976).

[11] M.A. Blanco, E. Francisco, and V. Luaña, Comput. Phys. Comm. 158, 57 (2004).

[12] K. Parlinski, computer code PHONON. Available at http://wolf.ifj.edu.pl/phonon

[13] R. Wahl, D. Vogtenhuber, and G. Kresse, Phys. Rev. B 78, 104116 (2008).

[14] W. Jeitschko, and A.W. Sleight, Acta Cryst. B 29, 869 (1973). 


\section{Tables}

Table 1. Ab initio calculated and experimental Raman frequencies $\omega\left(\mathrm{cm}^{-1}\right)$ for the $\mathrm{HgWO}_{4}$-type structure at ambient pressure.

\begin{tabular}{|c|c|c|c|c|c|c|}
\hline Mode & $\omega$ (ab initio) & $\omega$ (experiment) & & Mode & $\omega$ (ab initio) & $\omega$ (experiment) \\
\hline $\mathrm{B}_{\mathrm{g}}$ & 87 & 89 & & $\mathrm{~A}_{\mathrm{g}}$ & 353 & 374 \\
\hline $\mathrm{B}_{\mathrm{g}}$ & 133 & 139 & $\mathrm{~B}_{\mathrm{g}}$ & 491 & 508 \\
\hline $\mathrm{A}_{\mathrm{g}}$ & 141 & 145 & $\mathrm{~A}_{\mathrm{g}}$ & 525 & 537 \\
\hline $\mathrm{A}_{\mathrm{g}}$ & 184 & 193 & $\mathrm{~A}_{\mathrm{g}}$ & 683 & 698 \\
\hline $\mathrm{B}_{\mathrm{g}}$ & 200 & 204 & $\mathrm{~B}_{\mathrm{g}}$ & 695 & 715 \\
\hline $\mathrm{B}_{\mathrm{g}}$ & 216 & 228 & & $\mathrm{~B}_{\mathrm{g}}$ & 827 & 842 \\
\hline $\mathrm{A}_{\mathrm{g}}$ & 268 & 278 & & $\mathrm{~A}_{\mathrm{g}}$ & 907 & 924 \\
\hline $\mathrm{B}_{\mathrm{g}}$ & 280 & 292 & & & & \\
\hline
\end{tabular}




\section{Figure captions}

Figure 1. Ab initio calculated energy as a function of volume curves for the $\mathrm{HgWO}_{4}$-type (empty circles), wolframite (empty squares), $\mathrm{BaWO}_{4}$-II-type (empty diamonds), $\mathrm{Cmca}$ (empty triangles), $\mathrm{BaZnCl}_{4}$-type (filled circles), $\mathrm{LaTaO}_{4}$-type (filled squares), scheelite (crosses), $\mathrm{CaUO}_{4}$-type (filled diamonds), $\mathrm{BaMnF}_{4}$-type (stars), and $\mathrm{SrUO}_{4}$-type (plus signs) structures. The fergusonite structure was found to be identical to the scheelite, and the $\mathrm{CuWO}_{4}$-type to the wolframite. Energy and volume are written per formula unit.

Figure 2.(a) Volume as a function of pressure and (b) Gibbs free energy as a function of pressure. In (a) theoretical data for the $\mathrm{HgWO}_{4}$-type (circles), wolframite (squares), $\mathrm{BaWO}_{4}$-II (diamonds), and $\mathrm{Cmca}$ (triangles) is shown; crosses correspond to experimental data of the $\mathrm{HgWO}_{4}$-type structure. In (b) continuous lines are used for calculations at $0 \mathrm{~K}$ without zero-point motion effects, discontinuous ones for $300 \mathrm{~K}$. At each pressure and temperature the free energy is measured with respect to that of the $\mathrm{HgWO}_{4}$-type phase. Volume and free energy are written per formula unit.

Figure 3. Calculated lattice parameters $a, b, c$, and $\beta$ of the $\mathrm{HgWO}_{4}$-type (circles), wolframite (squares), $\mathrm{BaWO}_{4}$-II-type (diamonds), and Cmca (triangles) structures. Experimental data of the $\mathrm{HgWO}_{4}$-type structure is marked by crosses. For an easier comparison with the $P 2 / c$ wolframite, the $\mathrm{HgWO}_{4}$-type structure is shown in the $I 2 / a$ setting. The $c$ axis of the wolframite is shown multiplied by 2 . 


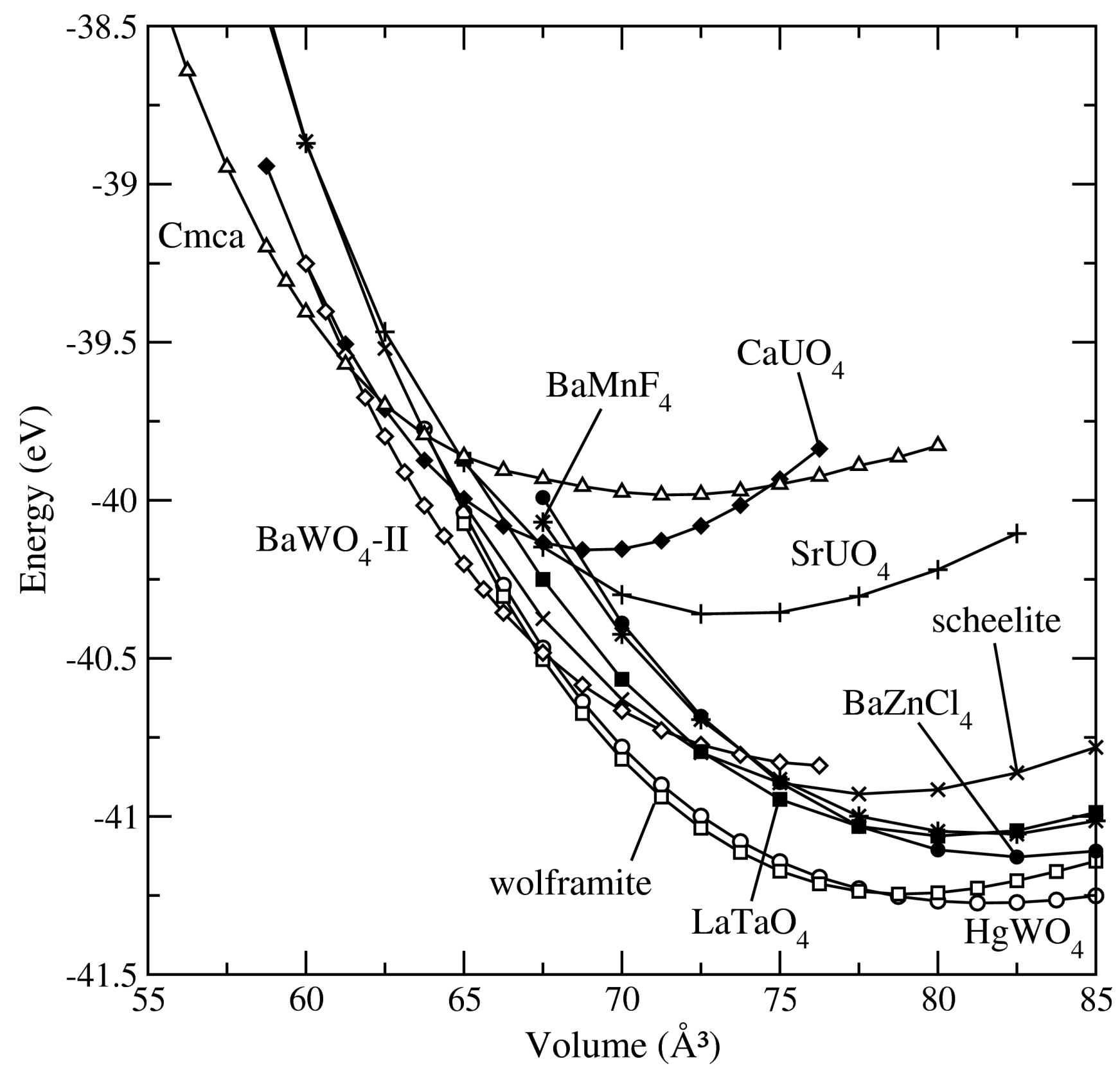

Figure 1. Ab initio calculated energy as a function of volume curves for the $\mathrm{HgWO}_{4}$-type (empty circles), wolframite (empty squares), $\mathrm{BaWO}_{4}$-II-type (empty diamonds), $\mathrm{Cmca}$ (empty triangles), $\mathrm{BaZnCl}_{4}$-type (filled circles), $\mathrm{LaTaO}_{4}$-type (filled squares), scheelite (crosses), $\mathrm{CaUO}_{4}$-type (filled diamonds), $\mathrm{BaMnF}_{4}$-type (stars), and $\mathrm{SrUO}_{4}$-type (plus signs) structures. The fergusonite structure was found to be identical to the scheelite, and the $\mathrm{CuWO}_{4}$-type to the wolframite. Energy and volume are written per formula unit. 


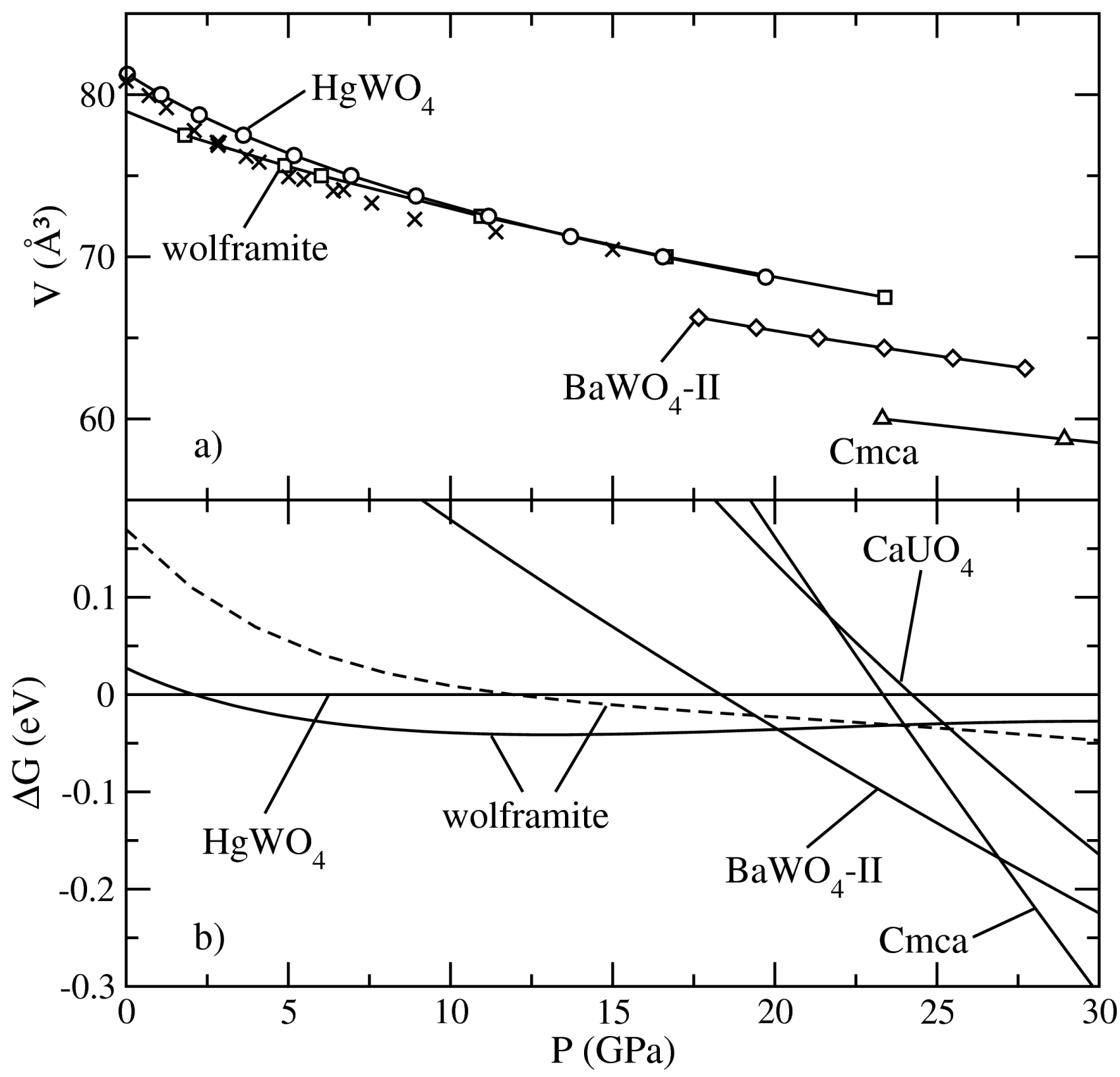

Figure 2.(a) Volume as a function of pressure and (b) Gibbs free energy as a function of pressure. In (a) theoretical data for the $\mathrm{HgWO}_{4}$-type (circles), wolframite (squares), $\mathrm{BaWO}_{4}-\mathrm{II}$ (diamonds), and Cmca (triangles) is shown; crosses correspond to experimental data of the $\mathrm{HgWO}_{4}$-type structure. In (b) continuous lines are used for calculations at $0 \mathrm{~K}$ without zero-point motion effects, discontinuous ones for $300 \mathrm{~K}$. At each pressure and temperature the free energy is measured with respect to that of the $\mathrm{HgWO}_{4}$-type phase. Volume and free energy are written per formula unit. 


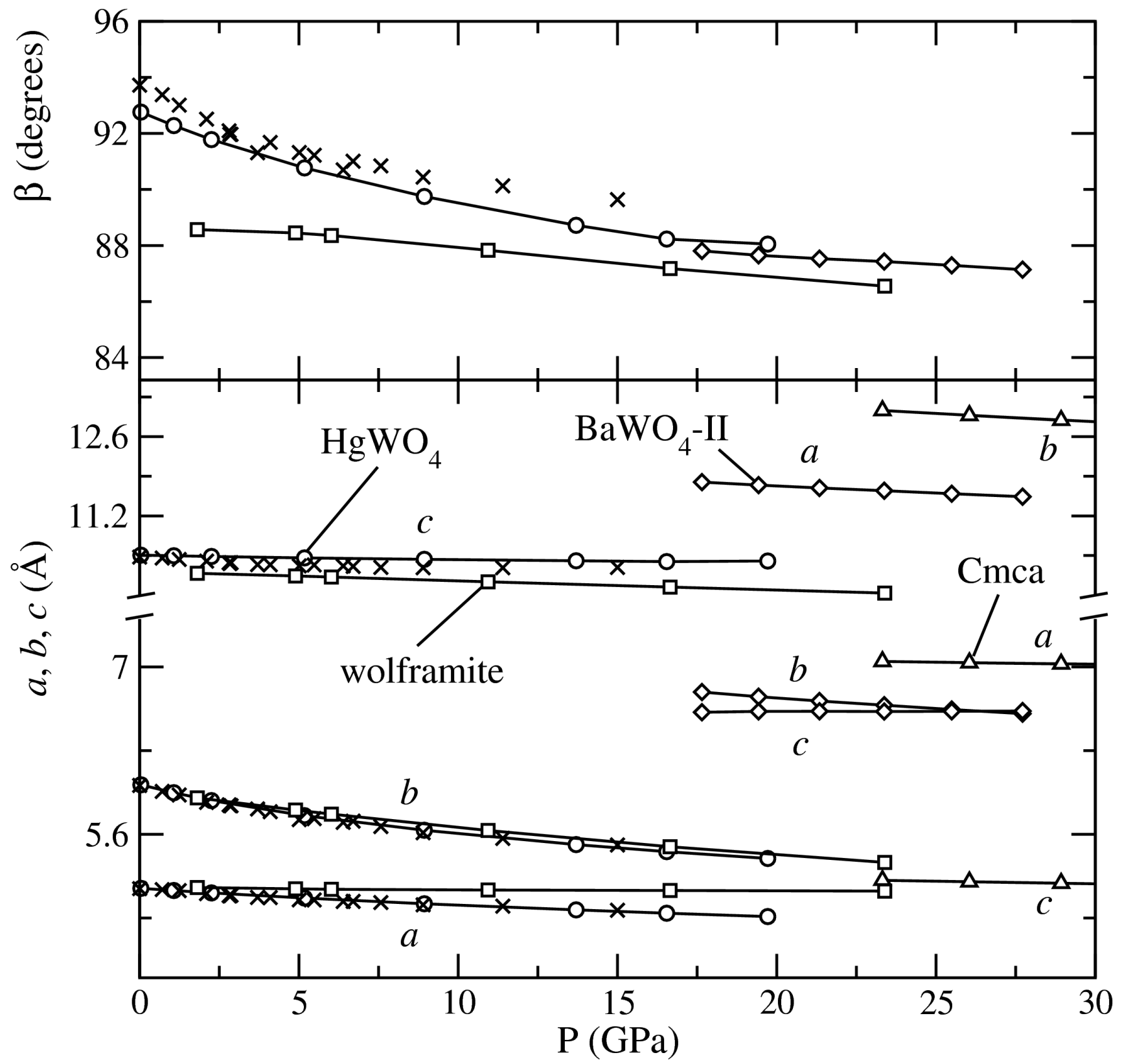

Figure 3. Calculated lattice parameters $a, b, c$, and $\beta$ of the $\mathrm{HgWO}_{4}$-type (circles), wolframite (squares), $\mathrm{BaWO}_{4}$-II-type (diamonds), and Cmca (triangles) structures. Experimental data of the $\mathrm{HgWO}_{4}$-type structure is marked by crosses. For an easier comparison with the $P 2 / c$ wolframite, the $\mathrm{HgWO}_{4}$-type structure is shown in the $I 2 / a$ setting. The $c$ axis of the wolframite is shown multiplied by 2 . 